\title{
高中数学教学中培养学生解决实际问题能力及数学应用意识的探 讨
}

左志广

新疆喀什地区第二中学

DOI:10.32629/jief.v2i11.2383

[摘 要] 在新时代的发展下, 高中数学教师在教学过程中, 要树立全新的教学观念, 从学生的数学思维入手, 进行高中数学教学模式的改革 和创新, 使学生解决实际问题能力和数学应用能力得到全面培养, 本文对在高中数学教学中培养学生解决实际问题能力及数学应用的意义进 行分析, 论述了培养学生解决实际问题能力及数学应用能力的教学方式。

[关键词] 高中数学; 解决实际问题能力; 数学应用

中图分类号: G633 文献标识码：A

在高中数学的教学中, 多数教师在课堂上最注重的是自己对数学知 识的讲解是否清晰, 而忽略了学生对于抽象知识的接受程度。所以, 教 师应将数学知识与生活实际进行联系, 让学生认识到生活中到处是数学, 激发学生对数学学习的兴趣, 提高学生解决实际问题的能力和数学应用 的能力。 \section{义 \\ 1 高中数学教学中培养学生解决实际问题能力及数学应用的意 \\ 1.1 可以提高学生对数学学习的兴趣 \\ 数学知识来源于现实生活和社会生活实践。所以。高中数学教师在} 教学中要呈现现实的、生活化的内容, 贴近学生的生活实际, 促进学生 对数学知识的理解, 体会到学习数学的价值, 对数学学习产生兴趣, 提 升学生学习的自主性。

1.2 可以改变传统的数学教学观念, 有利于素质教育的贯彻落实

在应试教育的影响下, 学生学习数学的主要目的为了高考的分数。 教师的教学也是为了满足学生的目的, 高中所有的数学教学内容在两年 之内教完, 课后进行漫长的题海战术, 使处于数学学习过程中的教师和 学生都非常痛苦, 最后的高考成绩却不理想。这说明脱离了实际应用的 数学教学的效果是必然不理想的。因此, 传统的教学观念的改变数学教 学的必然发展趋势, 在新的教学观念中学生数学应用的能力的培养是重 中之重, 这对于在高中数学领域推动素质教育的贯彻落实有着积极的促 进作用。

1.3 有利于教师自身专业能力的成长

在高中数学教材只有少部分的实际问题, 所以在培养学生解决实际 问题能力及数学应用能力时, 主要是依靠教师结合学生的实际情况, 创 造性地使用查询到的材料，对许多高中教师而言是一项不小的挑战。所 以, 教师在数学教学中要不断地学习, 丰富自身的知识储备, 先使自己 的数学应用能力和水平得到提高, 在进行教学, 在这过程中数学教师的 专业能力也会得到极大的成长。 效措施

2 高中数学教学中培养学生解决实际问题能力及数学应用的有

2.1 进行合理的课堂教学设计

在以往的高中数学教学过程中, 许多教师极少与学生进行沟通, 没 有根据学生的实际需求对教学内容和教学模式进行创新和改革。这样使 学生的数学思维和解决实际问题的能力都受到了禁锢。基于此, 为了培 养学生解决实际问题的能力及数学应用的能力, 高中数学教师必须要充 分把握数学中的重难点内容, 对教学内容进行针对性的调整, 采取有效 的教学设计。同时, 高中数学教师要充分考虑学生的思维发展特点, 从 学生的实际生活出发, 巧妙地在教学中引入学生熟悉的案例, 并在课后 为学生安排同样与实际生活相联系的练习环节, 培养学生解决实际问题 的能力和数学应用的能力。

\section{2 构建生活化数学教学情境}

高中数学教师要充分利用具体的生活场景来构建生活化的教学情 境, 让学生对于试题中描述的场景有足够的了解和认识, 激活学生对于 数学学习的情感, 引导学生进行数学问题的分析和思考。同理, 教师在
设计课后习题时，也要注意将习题与现实生活相关联。

例如, 可以编写一道与垃圾分类有关的问题: A 城和 B 城相距 $20 \mathrm{~km}$, 计划在两城外以 $\mathrm{AB}$ 为直径的半圆弧上选一点 $\mathrm{C}$ 建造垃圾分类中心, 垃圾 分类中心对城市的影响度与其到城市的距离相关，垃圾分类中心对 A、B 两城的总影响度为其影响度之和, 记 $\mathrm{C}$ 点到 $\mathrm{A}$ 城的距离为 $\mathrm{xkm}$, 建在 $\mathrm{C}$ 点的垃圾分类中心对 $\mathrm{A}$ 城和 $\mathrm{B}$ 城的总影响度为 $\mathrm{y}$, 统计调查表明, 垃圾 分类中心对 $\mathrm{A}$ 城的影响度与 $\mathrm{C}$ 点到 $\mathrm{A}$ 城的距离的平方成反比, 比例系数 为 4 , 对 $B$ 城的影响度与 $\mathrm{C}$ 点到 $\mathrm{B}$ 城的距离的平方成反比, 比例系数为 $\mathrm{k}$, 当垃圾分类中心建在圆弧的中点时, 对 $\mathrm{A}$ 城和 $\mathrm{B}$ 城的总影响度为 0.065 。 本题将理论知识社会热点相连, 使学生对这道题的解决方法产生了强烈 的好奇, 这道题对于现实生活中垃圾分类中心的建址也具有一定的指导 意义, 通过对本题的学习, 学生不仅会掌握更多的知识技能还会对垃圾 分类这一热点问题更加关注, 回应数学思维解决生活实际中的问题, 使 自己的解决实际问题能力及数学应用能力均得到提高。

2.3 开展有效的小组合作学习

因为学生个体对于数学知识的掌握程度不同, 解决实际问题的能力 不同。在数学课堂教学过程中, 如果面对全体学生用一种方式进行知识 点的讲解和分析, 会使学生整体得不到有效的数学效果, 甚至会导致班 级中学生数学能力两极分化现象的加深。基于此, 教师可以开展小组合 作教学模式, 将学生按数学学习能力的不同分为不同的合作学习小组, 并为每个小组安排不同的学习任务, 引导每一为学生在组内与同学进行 思考和讨论, 在学生完成了当前的学习目标后, 教师可以为学生设置难 度更高的学习任务, 这样分组分层进行教学的方式可以使不同层次的学 生都能够在解决实际问题能力及数学应用能力方面得到有效的提高。

\section{3 结束语}

综上所述, 学生解决实际问题能力及数学应用能力的培养对素质教 育的推进具有积极的正面意义, 在高中数学教学中, 教师可以通过在课 前进行合理的课堂教学设计, 并在授课过程中构建生活化的数学教学情 境, 最后开展有效的小组合作学习来提高学生解决实际问题的能力及数 学应用的能力。

\section{[参考文献]}

[1]张薇. 高中数学教学中提高学生问题发现与解决能力的策略 [ J] 数学教学通讯,2020(12):68-69.

[2]熊本超. 数学教学中培养学生解决实际问题能力的思考 $[\mathrm{C}$. 教育 部基础教育课程改革研究中心.2019年 “基于核心素养的课堂教学改革” 研讨会论文集.教育部基础教育课程改革研究中心:教育部基础教育课程 改革研究中心,2019:534-535.

[3]运熙伦.培养高中学生应用数学的教学研究 [C].北京中教智创信 息技术研究院.新课改背景下课堂教学方法与手段的有效性研究科研成 果集(第八卷).北京中教智创信息技术研究院:北京中教智创信息技术研 究院,2017:669-680.

[4]吴小明.关于提高学生应用数学解决实际问题能力的思考 [J].教 育教学论坛,2013(23):78-80. 